\title{
Título de la Publicación:
}

Competition between native Antarctic vascular plants and invasive Poa annua changes with temperature and soil nitrogen availability

\section{Autor:}

Cavieres, Lohengrin A., Sanhueza, Ana Karen, Torres Mellado, Gustavo, CasanovaKatny, Angélica

\section{Datos de Publicación:}

Biological Invasions, Vol.20, N6, 1597-1610, 2018

\section{Disponible en:}

https://www.scopus.com/record/display.uri?eid=2-s2.0-

85038078906\&origin=resultslist\&sort=plf-

$f \& s r c=s \& s t 1=C o m p e t i t i o n+$ between+native+Antarctic+vascular+plants+and+invasive+ Poa+annua+changes+with+temperature+and+soil+nitrogen+availability\&st $2=\&$ sid=cc 28cc7903b13e9935f677d7067ef777\&sot=b\&sdt=b\&sl=138\&s=TITLE\%28Competition+ between+native+Antarctic+vascular+plants+and+invasive+Poa+annua+changes+with+ temperature+and+soil+nitrogen+availability\%29\&relpos=0\&citeCnt=3\&searchTerm= 